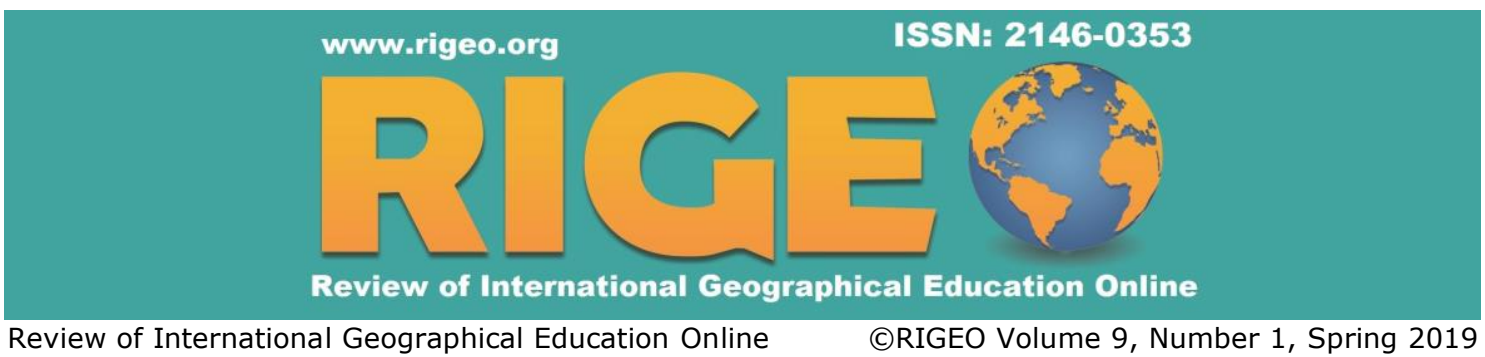

Research Article

Copyright $\odot$ RIGEO 2019

To cite this article: Cox, M., Elen, J., Steegen, A. (2019). Students' reasoning processes while constructing causal diagrams. Review of International Geographical Education Online (RIGEO), 9(1), 13-35. Retrieved from http://www.rigeo.org/vol9no1/Number1Spring/RIGEO-V9-N1-1.pdf,

DOI: $10.33403 /$ rigeo.573464

Submitted: August 21, 2018

Revised: January 31, $2019 \quad$ Accepted: February 17, 2019

\title{
Students' Reasoning Processes While Constructing Causal Diagrams
}

\author{
Marjolein $\mathrm{COX}^{1}$ \\ KU Leuven, Leuven, BELGIUM \\ Jan ELEN ${ }^{2}$ \\ KU Leuven, Leuven, BELGIUM \\ An STEEGEN ${ }^{3}$ \\ KU Leuven, Leuven, BELGIUM
}

\begin{abstract}
The use of causal diagrams to externalize the mental representation of a problem is recognized to be an important step in solving complex problems. In geography education several global challenges taught about in class are highly complex due to the interconnectedness of many causes and consequences. A systems thinking approach might be helpful to better understand these global challenges. Former studies have shown the effectiveness of concept maps and causal diagrams to foster students' systems thinking. However, it is not always obvious for students to construct proper causal diagrams. In order to optimize teaching strategies concerning these complex systems in geography education, this study analyzes students' cognitive strategies while constructing a causal diagram. We used task-based thinkaloud interviews to study their cognitive strategies. Four different cognitive strategies were observed. The different types of cognitive strategies all resulted in an acceptable constructed causal diagram by the students. The presented insights are explorative, but it reveals the thinking processes that are mostly tacit and therefore has the potential to contribute to better teaching strategies. After all, if we know what processes novices go through while carrying out a complex skill, which are often taken for granted by experts, in this case geography teachers, we can raise awareness among teachers to
\end{abstract}

\footnotetext{
${ }^{1}$ Corresponding author: Ms., KU Leuven, Faculty of Science, Department of Earth and Environmental Sciences, Celestijnenlaan 200E box 2409, 3001 Leuven, Belgium, marjolein.cox[at]kuleuven.be ORCID: 0000-0002-0805-7920

${ }^{2}$ Prof., KU Leuven, Faculty of Psychology and Educational Sciences, Instructional Psychology and Technology, Dekenstraat 2 box 3773, 3000 Leuven, Belgium, jan.elen[at]kuleuven. ORCID: 0000-0003-1611-5075

${ }^{3}$ Assoc.Prof., KU Leuven, Faculty of Science, Department of Earth and Environmental Sciences, Celestijnenlaan 200E box 2408, 3001 Leuven, Belgium, an.steegen[at]kuleuven.be. ORCID: 0000-0001-7011-2452
} 
explicitly take those processes into account while designing lessons.

\section{Keywords}

Cognitive Strategies; Reasoning Process; Causal Diagrams; Secondary Education; Systems Thinking; Geography Education; Think-aloud Interviews, Causality

Teaching and learning about complex systems is common in geography education (International Geographical Union, 2016). These complex systems often involve interaction between humans and their environment and are also characterized by the interconnectedness of multiple causes and consequences in a complex, rapidly changing world. A systems thinking approach, in which this complexity is taken into account, might offer a solution to better grasp the real complexity of global challenges such as international migration and climate change (Forrester, 1994; Senge, 1990).

Intervention studies in various domains using different teaching strategies have shown the possibility to foster students' systems thinking (Assaraf \& Orion, 2005; Hmelo-Silver, Liu, Gray, \& Jordan, 2015; Karkdijk, van der Schee, \& Admiraal, 2018). While an improvement of their systems thinking abilities can be observed, it is often not evident for students to adequately relate causes and consequences (Favier \& Van Der Schee, 2014; Karkdijk et al., 2018).

To optimize systems oriented teaching strategies and course materials, more insight is needed in students' cognitive strategies while performing exercises developed to enhance systems thinking. In this study in particular, we aim to acquire insight in students' reasoning while constructing a causal diagram to understand the system.

\section{Theoretical Background}

\section{Research on Complex Problem Solving}

Complex global challenges or problems such as climate change, hunger and migration flows are studied in geography. These problems can be considered to be complex ill-structured problems. Jonassen (2004) identifies four ways in which problems can vary: structuredness, domain specificity, complexity, and dynamicity. Solutions to ill-structured problems are not predictable, the procedure to solve the problem is often not known and concepts and principles from different domains are required to solve the problems. Furthermore, these problems are also highly complex and dynamic, due to the amount of involved interconnected variables and the fact that these variables, and thereby the problem as a whole, change over time (Jonassen, 2004). According to these criteria the problems mentioned above are ill-structured problems, also known as wicked problems. Complex problems require different intellectual skills and cognitive operations to deal with than well-structured problems (Funke, 2010; Shin, Jonassen, \& McGee, 2003), and therefore teaching strategies should be different (Jonassen, 2004).

Several authors describe cognitive strategies involved in complex problem solving. Weinstein and Mayer (1986), for instance, mentioned selecting important information in texts, relating content to already acquired knowledge, structuring the learning content in summaries and schemas, critically processing the content by forming an opinion about 
it, and applying the learning content as possible strategies for complex tasks. Dörner (1986 in: Fischer, Greiff, \& Funke, 2012) structured these strategies by distinguishing four different parts in the problem solving process: (1) information retrieval and integration to construct a model representing the system, (2) goal elaboration and balancing, in which the problem solver clearly identifies and describes the goals to achieve, (3) action planning and decision making to decide upon the strategies to apply and the kind of knowledge to rely on, and (4) self-management to manage time pressure, stress, frustration, and conflicts between inner values faced during the solving process. He emphasizes that the problem solving process should be looked at as a whole because the different parts are interacting with each other. Inspired by Dörner (1986), Fischer et al. (2012), separate two main phases in the solving process of ill-structured problems, namely (1) knowledge acquisition with a focus on information generation, information reduction and model building, and (2) goal-oriented knowledge application, with a focus on dynamic decision making and evaluation (Fischer et al., 2012; Funke, 2001). These two phases correspond to what Jonassen (2004) calls the two critical attributes of cognitive operations in complex problem solving. First, the problem solver must construct a mental representation of the problem and its context, also known as the problem space (Newell \& Simon, 1972). This problem space does not only contain knowledge on the structure of the problem, but also procedural knowledge and reflective knowledge (Jonassen \& Henning, 1996). Second, these mental representations or models need to be actively manipulated and tested by the learner.

In these lists of cognitive strategies to solve complex problems, all authors stress the understanding of the problem at stake as a first step. Research also provides evidence that experts are better problem solvers due to their ability to build more complete mental representations. In other words, the problem space in which the learner represents the problem seems crucial. To help learners in building a better mental representation, learners can externalize their internal problem via tools such as concept maps and model building (Jonassen, 2004). As these tools help to organize information, they can contribute to a better understanding of the related variables and to the elaboration of cognitive schemata in the head of the learner. These schemata can contain a lot of information, but are treated as one entity in a person's memory (Kirschner, 2002).

\section{Cognitive Activities during Modeling}

Externalizing mental representations and structuring knowledge about the complex problem via model building is an important cognitive strategy in complex problem solving, but this modeling process on itself requires several cognitive activities as well. Stratford, Krajcik, and Soloway (1998) distinguish analyzing, relational reasoning, synthesizing, testing and debugging, and explaining as the main higher-level thinking performances in which students are involved when creating dynamic models. Sins, Savelsbergh, and van Joolingen (2005) associate the following reasoning processes with modeling: analyze, inductive reasoning, quantify, explain and evaluate. The quality of these reasoning processes is different for more successful students compared to less successful students: successful students for instance look at the models as a whole instead of considering one relation at a time, use more prior knowledge and show more 
inductive reasoning. In general, they found that many novice modelers encountered serious difficulties in the modeling process and were therefore not able to use the model as a means to comprehend complex systems. Subsequently, appropriate support and scaffolding is emphasized. These findings are consistent with the study of Hogan and Thomas (2001) who revealed crucial differences between students in the phases of model construction, model quantification, model interpretation and model revision. The students who built the best quantitative ecological models, focused more on the behavior of the model as a whole when building and revising their model. These students always looked at the effect on the model output before adding or revising relationships within the model. Also Löhner et al. (2005) affirm that students may experience difficulties in a modeling task. They expected similar reasoning activities during model building as in the inquiry cycle (White, Shimoda, \& Frederiksen, 1999). These expected reasoning activities are orientation, hypothesizing, experimenting, and evaluating the model. However, they could not observe the inquiry cycle for the students in their study. The students spend most of the time on scientific reasoning, but not in a systematic temporal order (Löhner et al., 2005).

\section{Cognitive Activities during Concept/Causal Mapping and Solving Mysteries}

As an alternative to model building, more qualitative approaches are possible to structure knowledge and externalize mental models. A well-known tool is a concept map. This is a diagram in which concepts are spatially organized and the relations between these concepts are visualized by arrows accompanied with linking words (Novak \& Cañas, 2008). According to the theoretical model of McAleese (1994) the learner is engaged in a cognitive process at two levels during concept mapping; the operational level where the actions happen, often via a computer tool, such as creating or moving nodes, and the cognitive level where the actual thinking takes place. The operations can result from thinking, but will in turn encourage thinking as well. In the concept mapping process the learner represents concepts and makes claims or propositions about relations in the representation, viewing and checking phase. In a next phase, the resolution phase, ambiguities arise and the learner will experience mental conflicts which he has to solve. These viewing, checking, and resolving phases operate in a loop. In the final phase, called confirming, the learner for example expresses his agreement with the final representation (McAleese, 1994). Several of these cognitive activities are observed in the study of Dogusoy-Taylan and Cagiltay (2014), who compared concept mapping processes for experts and novices via eye-tracking. They identify constructing a concept, constructing a link, erasing, arrangement, reasoning, controlling and checking, cross-links, and revising as cognitive acts during the development of a concept map. Their results show that both experts and novices are involved in deductive reasoning, defined as 'starting with a general concept and continuing by constructing sub-concepts and links while keeping relations to the main concept in mind' (Dogusoy-Taylan \& Cagiltay, 2014: 87). But they also reveal differences throughout the entire process. For example, experts tend to complete an entire branch in the concept map first, instead of adding equal information to each subsequent branch. Moreover, the experts spend more time on constructing cross-links between branches, and showed less fixation as they tend to focus more on the concept 
map as a whole, instead of on particular elements (Dogusoy-Taylan \& Cagiltay, 2014). Finally, the authors emphasize regulation and awareness of the learning process as positively contributing to a better concept map and learning outcome (Bruillard \& Baron, 2000; Dogusoy-Taylan \& Cagiltay, 2014; McAleese, 1994).

In the study of Jeong (2014) the cognitive processes during the construction of causal maps were examined. Causal maps are a special kind of concept maps with only causal relations. The link between these relations are also visualized with arrows, and accompanied with a plus or minus sign instead of linking words (Öllinger, Hammon, von Grundherr, \& Funke, 2015). Their main findings suggest the iterative process of redirecting and deleting links in the maps as crucial in constructing causal maps. High performers revised their maps more often during the process (Jeong, 2014).

Finally, also the cognitive processes while solving mysteries are worthwhile to look at. A mystery is an exercise in which students solve a question, based on information on data strips. In order to solve the mystery they have to connect information of several data strips (Leat, 1998). Leat and Nichols (2000) were able to distinguish five different stages in the resolution process, which they also connect to the different levels of the SOLO (Structure of Observed Learning Outcomes) taxonomy (Biggs \& Collis, 1982). This SOLO taxonomy was developed to assess and classify students' learning outcomes in terms of their complexity. With a focus on the structural organization of students' responses to open-ended questions (the integration of several points or arguments into a structured response), they want to assess the quality of the response and distinguish mature thought from immature thought. It was developed as a reaction to assessing open-ended responses by counting how many arguments or points the students used in their response (Biggs \& Collis, 1982). In a first stage of the solving process of a mystery, the display stage, the students familiarize themselves with the data items. Some spread them out, others distribute them among the group members. This stage matches with the unistructural response of the SOLO taxonomy. In a second stage, the setting stage, students start to organize the data. The data strips are grouped based on common characteristics. This stage matches the multistructural response of Biggs and Collis (1982) in which data pieces are used but not strongly connected to each other. In the third stage, called the sequencing and webbing stage, the students are actually reasoning about relations between the data items. They start to find out partial explanations and are hypothesizing about them. According to Leat and Nichols (2000), this phase corresponds to the relational response in the SOLO taxonomy, in which several pieces of data are combined into a structured synthesis. Lastly, the students go through the reworking stage, in which data items are reorganized, and sometimes also the abstract stage, in which the students have internalized the data and keep on discussing on a more abstract level. These two stages fit the extended abstract responses in the SOLO taxonomy as a level of generalization and transfer is noticed (Leat \& Nichols, 2000). 


\section{Methodology}

\section{Research Design}

In this study causal diagrams are used as a mean to externalize the mental representation of a complex system and improving the comprehension of the system. The research described above clearly shows difficulties experienced by novices when constructing comparable representations such as concept maps or models. In this study it is examined what cognitive activities students show while constructing a causal diagram. This is done via students thinking aloud, as described below. By better understanding students' cognitive activities we hope to contribute to a further optimization of teaching strategies to handle complex systems, which are common in geography education.

\section{Sample}

In total 6 students out of two class groups of 16 students each, took part in the taskbased think-aloud interviews. To ensure diversity among the interviewees, their geography teacher selected the students taking into account differences in grades at school, cooperation during the lessons and general intelligence. The latter is on itself a widely discussed construct as many conceptualizations of intelligence exist (Esters and Ittenbach, 1999; Neisser, 1996). Some claim the idea that there is one overall factor of intelligence, while others are convinced that intelligence constitutes many different kinds of factors such as the nine factors defined by Horn \& Cattell (1966) including fluid reasoning, visual processing, a short-term memory. Many psychometric intelligence tests exists but are also critized for ignoring certain aspects of intelligence (Neisser, 1996). In this study the teacher is asked to estimate the overall intelligence of the students in order to choose students with a different intelligence level as we expect, based on cognitive load theory (van Merriënboer \& Sweller 2005), that students with a different intelligence level might use other strategies while constructing a causal diagram. This estimation is based on observations in the classroom and conducted geography tests for over a year.

All students are 17 years old and in the $12^{\text {th }}$ grade of the same school following the same course program with a focus on both natural and social sciences. The students have one geography lesson of 50 minutes each week. Most of the students in this course program will go to higher education after $12^{\text {th }}$ grade and about half of them will be able to graduate in a 3-year professional oriented bachelor program taught in a college. The students mostly do not start an academic bachelor program at univeristy (Ministerie van Onderwijs en Vorming, 2017).

\section{Students Thinking Aloud While Completing a Task}

Think-aloud interviews are based on the assumption that thinking is a sequence of thoughts, which can be verbalized without changing the sequence or content of these thoughts as well as the completion of the task (Ericsson, 2006). To prevent an altering of this sequence of thoughts, some guidelines have to be taken into account. First, research has found the highest validity if the subject verbalizes his thoughts immediately. Therefore it is important to create a nonreactive setting in which the 
subject feels comfortable to verbalize his thoughts and to practice thinking aloud in some warm-up tasks and to stimulate the verbalization if necessary (Ericsson \& Simon, 1998). Second, the interviewer should not ask why subjects responded in a specific manner. This could lead to a distraction of the task and alter the subjects' strategy. In fact, the interviewer should not interrupt at all to create this nonreactive setting (Ericsson, 2006; Ericsson \& Simon, 1998).

Protocol and data collection. Taking into account these guidelines a protocol was developed. The interview consisted of different phases: a welcome phase, a warming-up phase, the main phase in which the interviewee had to complete a task, a phase in which additional questions were asked about the content, a phase in which reflection on the task is stimulated and finally a phase in which the interview is closed.

During the main phase of the think-aloud protocol the students have to complete a task (Figure 1). First, they are asked to draw a causal diagram about the system behind the causes and consequences of cocoa cultivation in Côte d'Ivoire. They receive information in the form of texts, figures and maps. Furthermore, the variables that could be used in the diagram were provided for the students on the assignment sheet, but also separately on paper strips. During this phase it was very important that students would reason out loud. If the interviewer noticed the students not reasoning out loud anymore, the student was encouraged to think aloud again. Second, once they had drawn the diagram, they were asked to explain the system as if they would do to pupils in primary school. 


\section{Part 1:}

Study the information sources about the cocoa cultivation in Côte d'Ivoire.

\section{Clarify with a causal diagram the system behind the causes and consequences of the cocoa production in Côte d'Ivoire.}

Use all variables mentioned below in your causal diagram, You receive these variables also on paper strips. You can choose yourself if you use these paper strips. You make as many notes, drawings, summaries,... as you like. Please, draw the causal diagram on the foreseen squared paper.

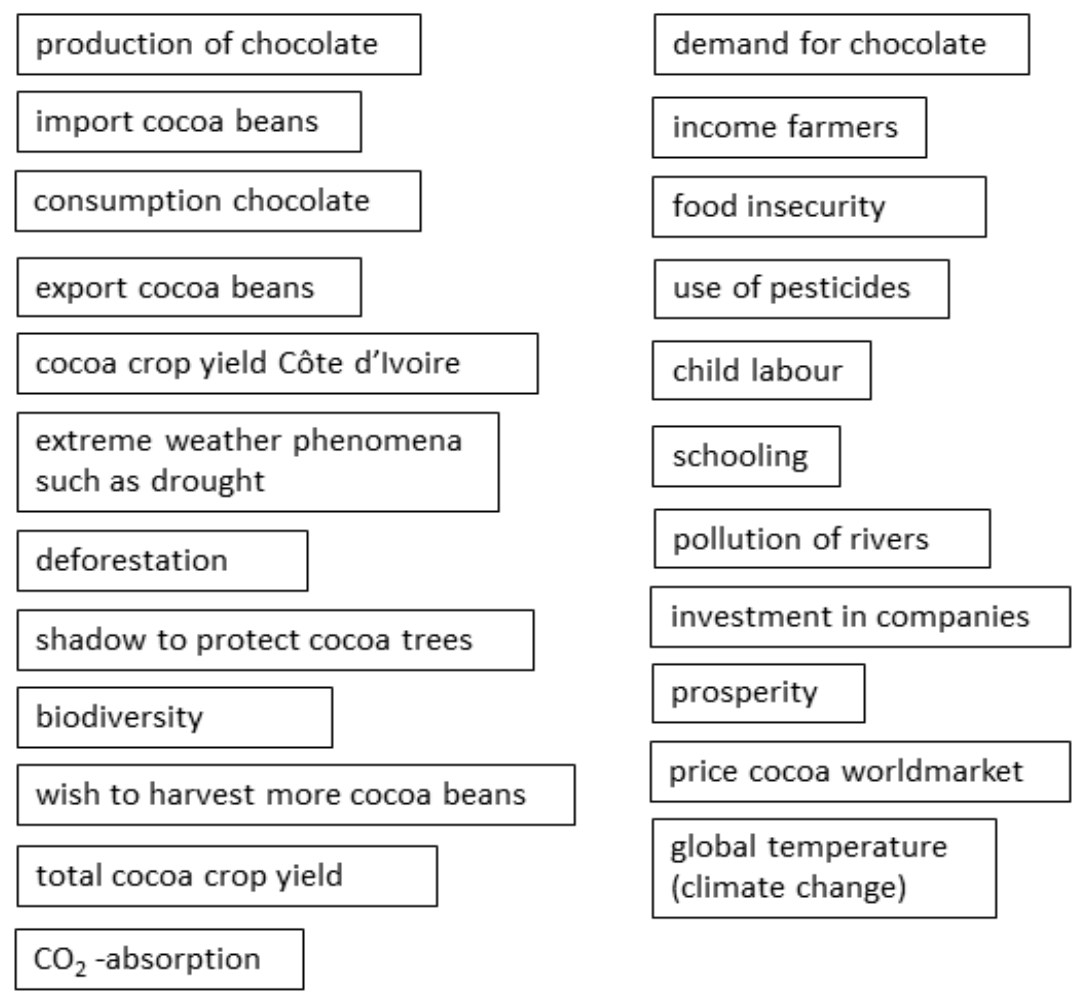

\section{Part 2:}

Explain the problem as you would to this for a group of pupils from primary education. You can use the paper to make notes or a scheme.

Figure 1. The task to be completed by the students while thinking aloud. The information sources the students have to read as part of this task are not included, but available with the authors upon request.

In the reflection phase the students were asked to reflect upon their own strategy and their geography lessons. Questions were asked such as 'Would you do things 
differently? If yes, which things would you do differently and why?' and 'Do you think the current geography lessons are useful to process geographical learning content?'

The entire interview lasted approximately 1 hour and was audio- and videotaped. The notes taken by the students were recorded with a smartpen. This smartpen looks similar to a normal pen, but has an audio recorder and a minuscule camera inside which records the notes and is able to connect the written notes to what is said at the exact moment a particular note is taken. The interviewers were three researchers, who were all trained in order to keep the conditions across the different interviews as similar as possible.

Analysis. The interviews were transcribed and coded descriptively in NVivo 11. This software enables to select and code parts of transcripts, texts, spoken language, images and films. These labeled fragments can then further be qualitatively analyzed through categorization, organization and visualization. In this study the transcripts and the diagrams drawn by the students with the smartpen were at the center of the coding and analysis in Nvivo. This coding was blind in the sense that the researcher did not known to which student each transcript belonged to. In this descriptive coding segments, defined as parts of the transcript consisting of one cognitive activity, were labeled to distinguish between different kinds of cognitive activities e.g. reading out loud a text, paraphrasing the information in the text, describing a figure, reasoning while constructing the diagram, explaining the diagram, thinking about the strategy, responding to the questions in phase 4, responding to the questions in phase 5, and so on. During this coding process the videotapes as well as the audio tapes and registered notes of the smartpen were used to optimize the interpretation of transcriptions.

The coding process consisted of two phases in order to obtain a good inter-rater reliability. First, two raters separately coded one interview without any provided codes. Afterwards the two raters discussed the codes and agreed upon a set of codes to be used in the next phase. Second, all interviews were coded with the agreed upon coding tree. One randomly chosen interview was coded by both raters to calculate the inter-rater reliability, which resulted in a high average agreement of 97\%. The Cohens Kappa, which takes into account the possibility of agreement by chance, is 0.64 , which is substantial according to Landis and Koch (1977).

Once the transcripts were coded, they were further analyzed to better understand the students' cognitive strategies. To do this in a systematic way a list with questions was developed, structured in line with an operational definition of systems thinking (Figure 2). Drawing and explaining causal diagrams are two of the three skills defined in the operational definition (Cox, Elen, \& Steegen, 2017). In the same table the responses to questions regarding the reflection on the task and teaching strategies were included. The transcriptions, observations, and coded segments were used to answer these questions. Results for each question were put in a large table in Excel with a column for each participant to facilitate the comparison between the strategies of the different students. 


\section{Questions concerning the operational definition of systems thinking:}

1. Constructing a causal diagram based on information of a given source

\section{a. Identify relevant variables}

i. Does the student use all the provided variables in the drawn diagram?

ii. If the student does not use all the variables in the diagram, are these variables mentioned during the reasoning process? What is said about these variables?

iii. Where does the student start to construct the diagram?

iv. Remarks (e.g. in case the student adds extra variables)

\section{b. Recognize relations between variables}

i. Does the student experience difficulties to recognize specific relations? Which relations are difficult?

ii. Does the student use own prior knowledge to explain relations in addition to the provided information? If yes, when?

c. Assign the nature of these relations ( + or -$)$

i. When does the student assign the nature of the relations? (e.g. immediately if he draws the arrow, after all the arrows are drawn,...)

ii. Does the student experience difficulties doing this? Explain.

iii. Remarks

d. Questions regarding the cognitive strategies related to all three subskills (a, b and c)

i. Which sequence of actions does the student undertake to construct the diagram?

ii. What is the first action of the student after reading the assignment sheet?

iii. In what order does the student read the provided pieces of information?

iv. Does the student read all the information sources? If not, which are not read?

v. Does the student indicate or mark things in the sources? What?

vi. Does the student take notes? What does he/she write down?

vii. Are there actions on which the student spend remarkably more time?

\section{Describing relations between variables in words}

i. Does the student experience difficulties in explaining the diagram?

ii. Does the student adjust the diagram while explaining it?

\section{Questions regarding students reflections about their strategy and the teaching} strategy in class:

i. What would the student do different the next time if any?

ii. Opinion about current teaching strategy in geography class.

Figure 2. Questions answered for each think-aloud interview to systematically analyze these interviews regarding the research question. The structure of the questions is based on the operational definition of systems thinking, with elements in bold being literally mentioned in the operational definition of Cox, Elen and Steegen (2017).

\section{Findings}

The results are structured following the questions in the table above, which is mainly reflecting the operational definition of systems thinking adopted in this study. 


\section{Constructing a Causal Diagram: Identifying Relevant Variables}

Of the six students, five use all the provided variables in their final diagram. One student does not use two of the 23 provided variables. In an early phase she mentions that she does not know where to place one of the variables, namely 'investment in companies'. She puts the paper strip with the variable aside. In a later phase, when most of the variables are related to each other, she returns to strips set apart. She reasons about one of the two which she is able to relate to the other variables and places it in the diagram. At this time only one variable seems to be lacking in the diagram. No reasoning about the other variable is observed anymore. She leaves the paper strip on the page without drawing any connection to the other variables. When she actually writes down the variables and draws the connections, which is mostly writing down the variables that are on the paper strips and redrawing the connections, she does not mention the variable anymore. It gets lost between the other paper strips. The second variable which is not in her final diagram, was actually placed on the diagram with the paper strips and arrows were drawn with other variables. It is not clear why she does not copy it on her final written diagram. Furthermore, none of the students adds variables to the diagrams in addition to those provided in the assignment sheet. In the assignment sheet adding additional variables was not suggested, but it was not prohibited either.

Regarding the variable they use as starting point to draw the diagram, there is a remarkable similarity. Four students choose for the variable 'demand for chocolate'. Two other students start their diagram with 'use of pesticides'. This similarity might be partly explained by the order of the provided information pieces. The students who start with 'use of pesticides' as a first variable in the drawing of the diagram probably do this, because this variable is focused upon in the first piece of information. They start drawing their diagram quite early in the process and have not yet developed an overview.

\section{Constructing a Causal Diagram: Recognizing Relations}

Overall the system is quite well understood by the students and no big difficulties were observed concerning the identification of relations. However, sometimes relations are drawn that are odd at first sight. This is due to the interpretation of variables, which is sometimes different than the authors intended to in the design of the assignment. For example, the variables 'production of chocolate' and 'cocoa crop yield' are sometimes confused by the students. The fact that the word 'cocoa production' is used in one of the provided maps in the task might have contributed to the confusion. Another example is the variable 'investment in the company' which seems to be more associated with the 'production of chocolate' instead of with the 'agricultural company/ the farm':

"One more thing and that's the thing about investment, but where does this belong to? Investing in a company. One needs more production, which probably leads to the need for more investment because otherwise you don't have enough chocolate.... But hey wait, those local farmers can't invest in an expansion or something else, because they don't have the money for it." She reads part of a text in which the variable investment in a company is actually included and says: "So, that is a bit like starting a company." 
Cox, M., Elen, J., Steegen, A. (2019) / Students' reasoning processes while constructing causal....

Later on while drawing the arrows she reasons: "There has to be more production of chocolate.... Investing in a company, and so this is... investing in a company, production, that's a plus." (Student 1)

\section{Constructing a Causal Diagram: Assigning the Nature of Relations}

Four of the six students are well able to assign the nature of the relations. In their reasoning it is clearly observed that they take time to reflect about which sign they have to add. Two examples:

"Due to deforestation, due to deforestation the shadow protecting the cocoa beans will also decrease, so there is more deforestation and less shadow, so that's a minus. Harvest of the cocoa trees in Côte d'Ivoire will also decrease because of extreme weather phenomena. That's a minus." (Student 1)

"Consumption of chocolate, consumption of chocolate is going to increase which will lead to an increasing demand for chocolate, so that's a plus. The production of chocolate will also increase because of this, so that's also a plus." (Student 2)

One of the other two students experiences assigning the nature of the relations as a difficult part of the task. In the beginning he starts assigning a plus or a minus, but he does not seem to reason in a systematic way, it seems more of a guess:

"So, this leads to a plus for pollution of rivers and also, oh no wait, that should be a minus than, oh, how did this work again with those plus and minus signs? Should I really add these plus and minus signs? [...]I'll take a minus, or no a plus. And these two also lead to less biodiversity, yes, or only this one? Wait, I'll write it here for a moment, biodiversity.... It is also possible that the pollution of this rivers, yes this also causes less biodiversity because they drink from the water of course. So, these are already two consequences. Yes, a plus, a minus. I'll take a minus."

After a few relations he decides to wait with adding a plus- or minus sign until he has drawn all the relations. Once he has reached this phase, he adds a sign to each arrow, but the reasoning behind it is not clear as he quite quickly goes over it and he does not really express his thinking at that moment. Several mistakes are observed in the final diagram.

Another student does not use plus- or minus signs in her diagram. Therefore, it can hardly be called a causal diagram. However, she does reason whether an effect will be increasing or decreasing, but she writes this down using a small increasing or decreasing arrow. Figure 3 shows an excerpt of the diagram, which is accompanied with the following reasoning:

"Euhm because of all these things it will have many consequences on a global scale, more in particular $\mathrm{CO}_{2}$-absorption will decrease, biodiversity, this will decrease too. Global temperature, temperature will also, euh... it will increase. Euh, and extreme weather phenomena, extreme weather phenomena this will happen more often. " (Student 6) 
This kind of notation is not preferable in a causal diagram as it hinders the understanding of changes in a system if variables increase or decrease due to an interference in the system.

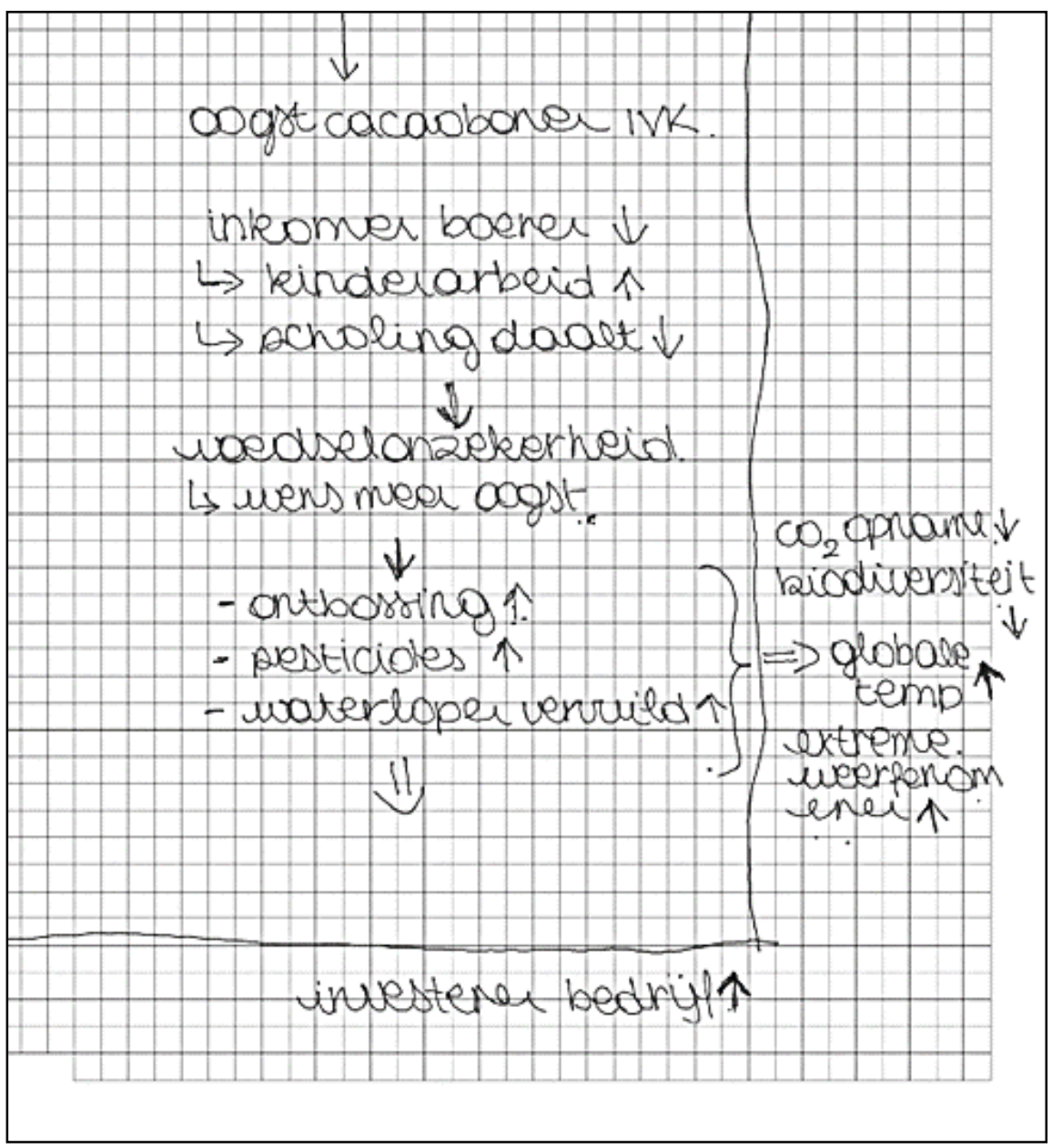

Figure 3. Excerpt of the diagram drawn by student 6. She does not draw a real causal diagram, but uses small arrows that go up or down to show the nature of a relation.

\section{Cognitive Strategies to Construct a Causal Diagram}

Concerning the students' overall cognitive strategy, consisting of a sequence of cognitive activities, four different types could be distinguished (Figure 4): a) linear strategy, b) a recursive linear strategy, c) a grouping first recursive linear strategy, and d) a group and identify recursive linear strategy. In all these strategies students start with reading the instructions of the task at hand. The students all read the content materials, identify variables, relate variables and have a review and reflecting phase in the end. However, the sequence of these activities varies for the different types. 
Cox, M., Elen, J., Steegen, A. (2019) / Students' reasoning processes while constructing causal....

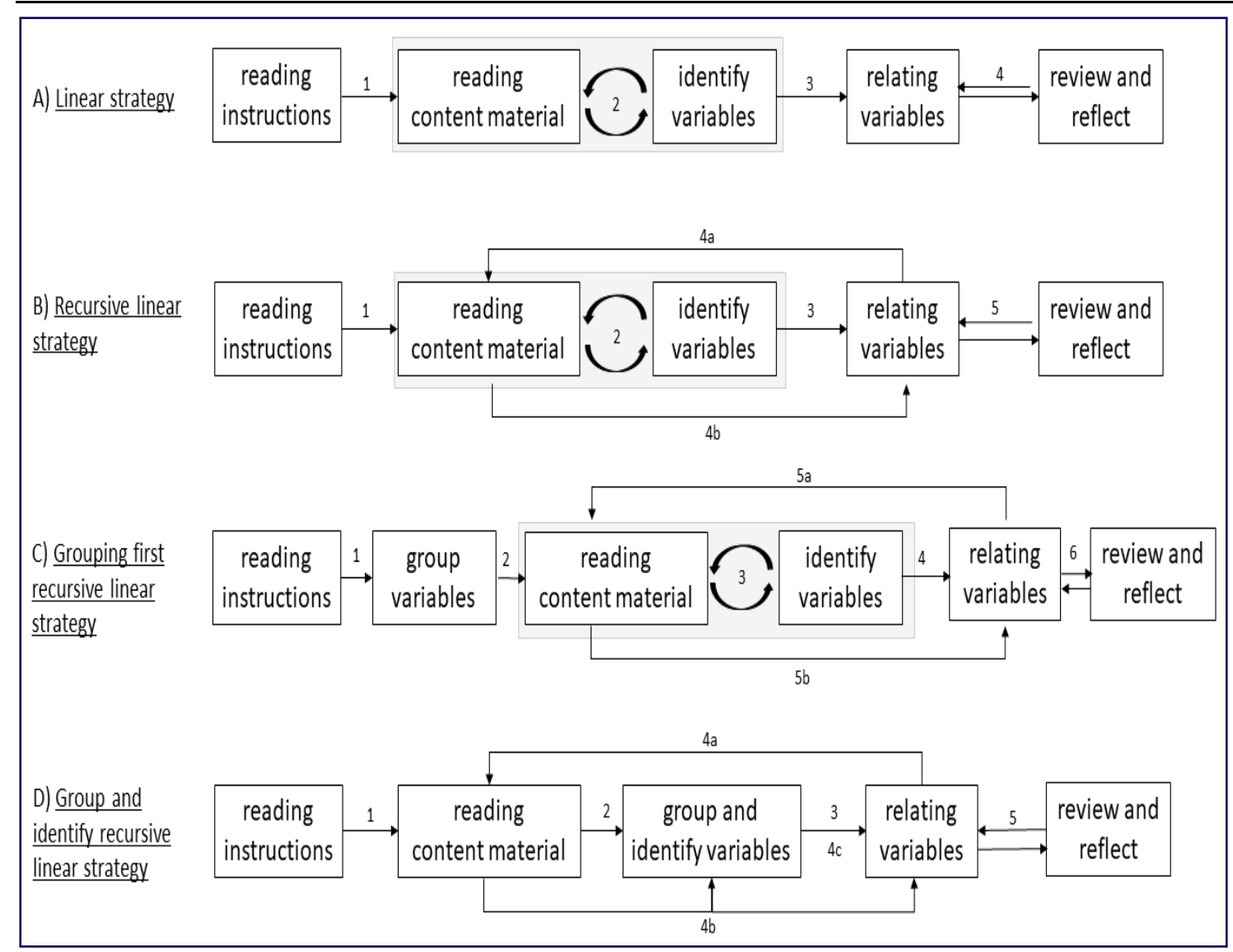

Figure 4. Different types of cognitive strategies used by the students to construct the causal diagram based on provided content material. The numbers indicate the sequence of the steps in the process.

Two students follow a linear strategy. After reading the instructions, they start to read the content materials. For each information source they identify variables. The curved arrows in step two visualize that variables are identified for each piece of information, before the following piece of information is read. After reading the information and identifying the variables, they start relating the variables and drawing the diagram. Specific for the students in this type is that they almost never consult the content materials anymore once they start reasoning about the relations and draw the diagram. After drawing the diagram they check whether it is complete. Within this type there are two observed variants. In the first variant the student puts the paper strips with the variables identified in the information on the printed information source. In this variant the student also starts drawing the diagram immediately after the identification phase. In the second variant, the student writes a brief summary for each information source with the variables identified in this source. This student also draws the diagram with the variables on paper strips first before writing it down afterwards. A second type of cognitive strategy is the recursive linear strategy. In this type the student starts with reading the instructions and the content materials. The student identifies variables for each piece of information, but while constructing the diagram the student often goes 
back to the content materials. He rereads parts of the information before drawing arrows and connecting variables.

A third strategy is called the grouping first recursive linear strategy. The student who follows this strategy reads the instructions after which she takes the variables on the paper strips, spread them on the paper, and tries to group the variables based on prior knowledge (Figure 4). After grouping the variables the student reads the content materials, identifies variables from the grouped paper strips for each read piece of information, and start relating the variables into a diagram. Finally, the student quickly reviews the drawn diagram.

The fourth strategy is the group and identify recursive linear strategy, followed by two students. After reading the instructions the students read all the content materials. After reading all the information they take the paper strips and try to group them, for example variables related to demand are put together, variables related to consequences are put together and so on. They start from these grouped variables to identify a variable and start drawing connections between them. In the phase of relating the variables they often reread pieces of the content materials and select variables. Afterwards the students review and reflect briefly upon the drawn diagram.

Although the students use different overall strategies to construct the diagram, they all use the paper strips in their reasoning process. Some use it to draw the entire diagram (Figure 5), while others use them to identify variables in information sources and link them to it (Figure 6).

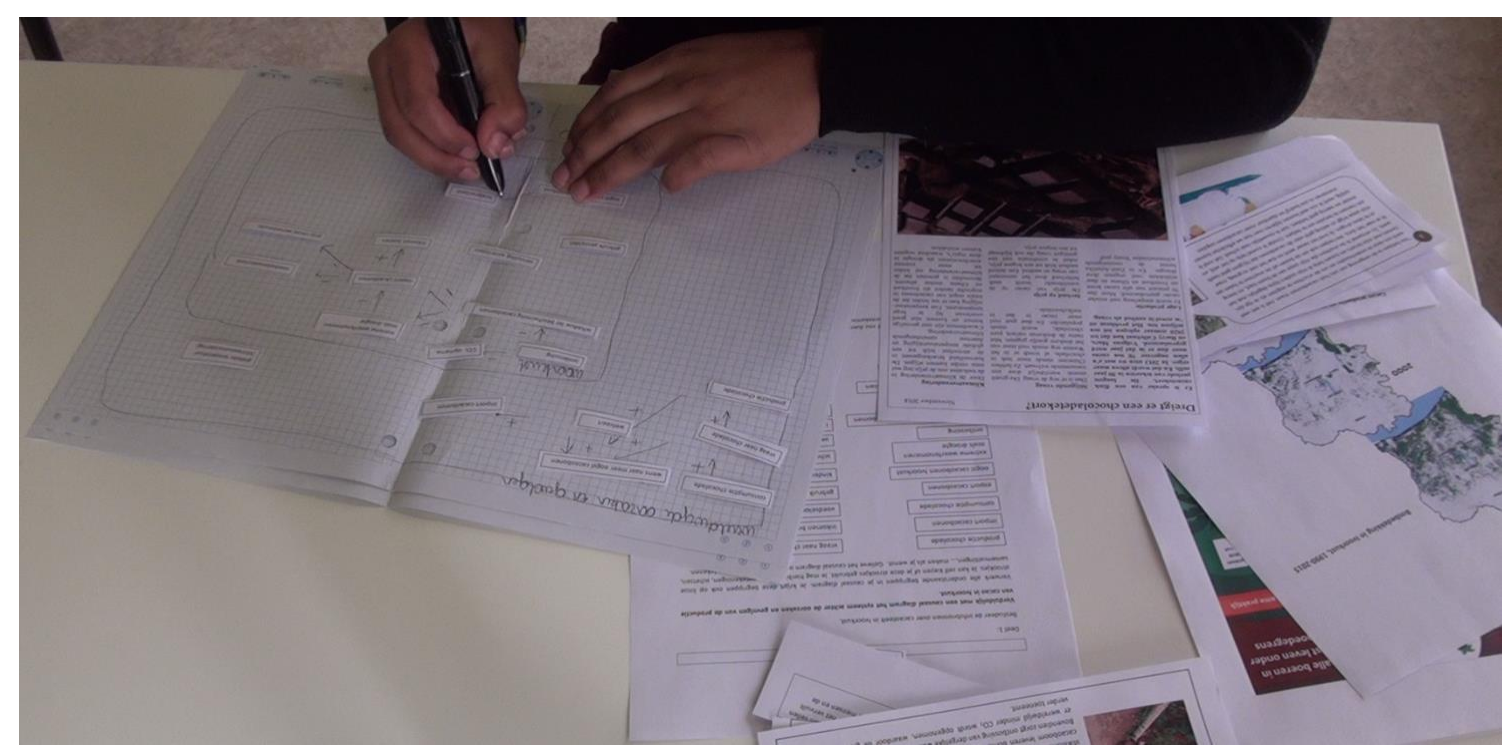

Figure 5. The student uses the paper strips to construct the causal diagram 


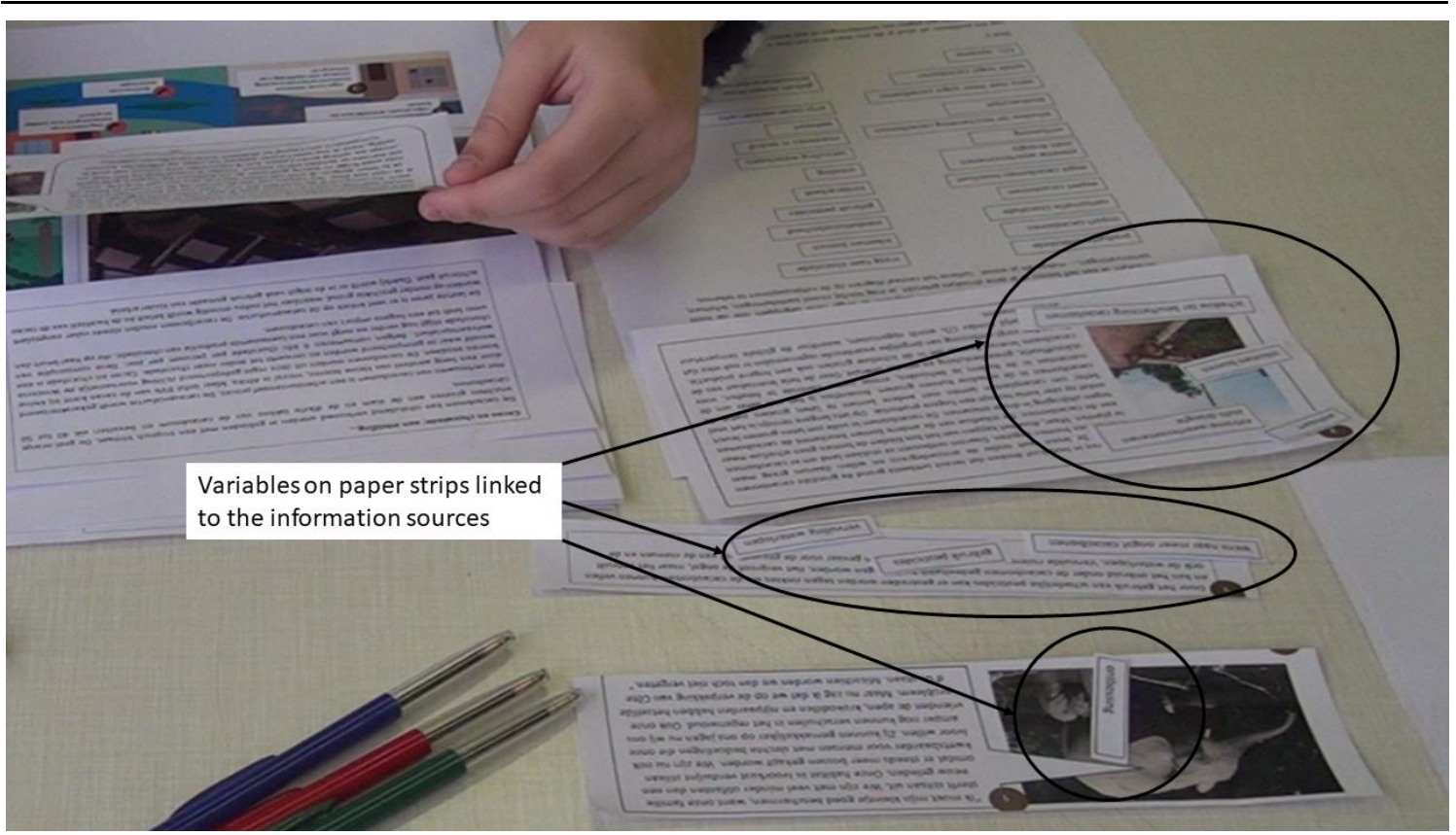

Figure 6. The student identifies variables in each source of information and put the paper strips on the information in which the variable is identified.

Furthermore, overall the students stick close to the information provided in the sources to relate variables. Only exceptionally they seem to use their own prior knowledge while relating variables when they do not succeed in finding the necessary information in the information sources.

\section{Describing Relations between Variables in Words}

In the second part of the task students explain the drawn diagram. It is observed that students quite well understand the system. Sometimes their explanation of the systems and its relations is not very well expressed but it is clear that they understand what is going on with cocoa cultivation in Côte d'Ivoire and the global chocolate consumption. While explaining, some students notice lacking arrows or wrong signs added to an arrow, and adjust these. In general, students do not seem to experience real difficulties in literally describing their diagram. Although much progress is possible in terms of bringing a coherent story about the system with a better structural organization of their explanation.

\section{Students' Reflections about Their Strategy}

When reflecting about their own strategy, two students are quite satisfied. However, one student mentioned to reread some of the information sources while drawing the diagram. The other one talked about making a list with causes and consequences while reading the information sources.

Two other students were completely satisfied with their approach and would do it exactly in the same way the next time. The last two students mentioned some practical concerns. They started drawing the diagram rather early and would use the paper strips more intensively next time to spatially organize their diagram before writing it down. 
They expressed the feeling to lose the overview a bit and were concerned about drawing a chaotic diagram:

"... maybe I would try to relate more with the paper strips, before I start drawing, because now I only started to relate and draw these relations on paper, but this is not very neat, and probably I missed a few things. So it would help to have a better overview or so." (Student 3)

\section{Discussion}

In the task-based think-aloud interviews all the students achieve to construct an acceptable causal diagram and show a rather good understanding of the system behind the cocoa production in Côte d'Ivoire. The students' cognitive strategies differ but are all quite successful to gain insight in the studied system.

\section{Interpretation of the Cognitive Strategies While Mapping}

The cognitive strategies observed are similar to those found in literature about concept mapping or modeling. In the phases we called identifying variables, relating variables and reviewing, the students also make propositions about relations, encounter ambiguities and solve them as is the case in the viewing, checking, and resolving phase of McAleese (1994). As we have illustrated in Figure 3 most of the students go iteratively through these phases, while rereading the information and reviewing the relations between variables which is also consistent with McAleese (1994), who states that these phases operate in a loop. The importance of this iterative process was emphasized as well by Jeong (2014) who found high performers revising their maps more frequently throughout the process. Furthermore, several of the stages observed during the solving of a mystery, could be recognized here as well, such as the display and setting stage in which students familiarize themselves with the data and start to organize the data (Leat \& Nichols, 2000). Despite the fact that the students do not really formulate one hypothesis, these phases might be compared to the orientation and hypothesizing phase of the inquiry cycle (White et al., 1999), certainly for the student of type $\mathrm{C}$ with a grouping first recursive linear strategy, who groups the variables beforehand based on prior knowledge. Also the sequencing and webbing stage, which is called relating variables in our study, is observed for all 6 students, as is the reworking stage, called review and reflect phase. When comparing these stages to the SOLO levels, at least all the students achieve the relational response, in which several pieces of data are combined into a structured synthesis (Biggs \& Collis, 1982; Leat \& Nichols, 2000).

As the focus of this task was on conceptually reasoning about the relations between variables in a system, it is of no surprise that cognitive operations typical for computer modeling, such as testing (Stratford et al., 1998) and quantifying, are not observed. A causal diagram represents a conceptual model of the system, but is not made to simulate the system as there is no quantification involved. On the other hand, studies on modeling show novice students encountering serious difficulties in the construction of the model which impede them to use the models as a mean to comprehend complex systems. By drawing causal diagrams on paper, some of these difficulties are 
eliminated, while still fostering relational reasoning. Modeling has the potential to achieve deeper learning and testing hypothesis via the simulation possibility, but this study shows that drawing causal diagrams might be used as a first step for novices, or even as the preferable step in secondary education, before being engaged in computer modeling in higher education.

\section{Findings to Integrate in Future Instructional Design}

The findings combined with the acquired knowledge concerning the cognitive strategies already revealed some important elements to take into account in future interventions, both from a conceptual and from a practical point of view. From a conceptual perspective it can be discussed how teachers can invest this acquired knowledge on cognitive strategies into their lesson design. Apart from externalizing the mental representations, it is also useful to clearly mention and externalize the cognitive processes students should go through while performing a task. According to the idea of cognitive apprenticeship these cognitive activities are often tacit in current schooling, and used to be more explicit and observable for the learner in ancient times where traditional apprenticeship was the natural way of learning, even before schools existed (Collins, Brown, \& Newman, 1989; Collins, Brown, \& Holum, 1991). Now that the cognitive strategies are known, they can be shown or even discussed in the classroom to improve students' procedural knowledge in solving complex problems, and hence broadening the problem space (Newell \& Simon, 1972). More in particular this knowledge on students cognitive strategies can be used in at least three of the six methods proposed by Collins et al. (1991) to design learning environments for cognitive apprenticeship. These three methods are modeling, coaching and scaffolding. The modeling method is about the demonstration by the teacher. The learner observes the teacher completing a task and tries to think about the different steps that are undertaken by the teacher. These steps often involve cognitive activities not visible for the observer. In order to enhance the learning process it is important that the teacher explicitly expresses these cognitive activities. However, an important condition is the teachers' awareness about these cognitive strategies. Otherwise the teacher will not be able to clearly name and distinguish the different cognitive activities. Knowledge on these strategies can also enable the teacher to explain different alternative strategies to the students. Also in the coaching and scaffolding method the teacher can use the acquired knowledge. It will help to design instructions and course materials with the right level of scaffolding. Also during the lesson teachers might be able to support students with more appropriate and direct feedback since they might detect problems sooner once they know in which cognitive activity students are involved.

From a practical point of view, three observations are worth considering into future design. First, offering paper strips with the variables seems very valuable to foster the relational reasoning of students. It was observed that all the students intensively used them, and the students who used them only in an early phase expressed to also use them while spatially organizing their diagram if they would have to do the task again. It creates the possibility to hold an overview on the system. When the students are working with these paper strips the mutual stimulation of cognitive processes on the operational and the cognitive level, as stated by McAleese (1994) is clearly observed. In 
other words, moving variables is a result of thinking, but also initiates thinking processes. If in the future more complex tasks are included in the lessons, students can also identify the variables themselves and write them down on adhesive notes for example, or use a software to construct causal diagrams which also offers the possibility to create, delete and move variables. Second, a careful use of language in provided sources and variables was noticed as important throughout the reasoning process. The interpretation of texts and variables might be different from what is intended, which can eventually lead to different relations. As students stick quite close to the information in the provided sources it seems important to carefully think about the words used in these sources. On the other hand, the ultimate goal is for students to search for information themselves. This information will not be developed for students to understand the system and will not contain these relations as explicit as is the case in the designed information sources provided in the task. As already mentioned future research is needed to study how this complexity can be gradually developed. Third, the observation that several students start with the same variable to construct the diagram highlights students' need for a kind of 'starting point' in their reasoning process. Perhaps, teachers can spend attention to this if they are coaching students who are struggling constructing causal diagrams, but it might also be an option to include this starting point in the research questions that guide the students.

\section{Challenges for Future Research}

Due to the limited number of students involved in this explorative study we were able to qualitatively analyze the cognitive activities students go through while constructing a causal diagram. This led to four different types of cognitive strategies as described in the results. However, it is clear that more research is needed to test the prevalence of these cognitive strategies in large-scale studies. If many students are involved it is possible to take into account student characteristics to see which strategy works best for who, and to study whether some cognitive strategies might be more successful compared to others. Further research would also create the possibility to study whether these cognitive strategies are still present in more complex tasks, and in tasks were variables are not provided. This task should be interpreted as an exercise in the construction and externalization of a mental representation in order to foster a deeper understanding of a complex system. Indeed, different authors emphasize gaining knowledge about complex systems via information retrieval and integration to build a model, schema or a mental representation, as a first important step in the problem solving process (Fischer et al., 2012; Jonassen, 2004; Weinstein \& Mayer, 1986).

\section{Conclusion}

This study provides a deeper insight in students' cognitive activities while constructing a causal diagram based on given variables and information sources. Different cognitive strategies all led to an acceptable understanding of the complex system. Despite the need for further research as suggested in the discussion, it offers the potential to take this knowledge into account in future teaching strategies. Based on our current insights the use of causal diagrams as a tool to visualize complex systems can be argued to deserve a place in geography education as geography deals with challenging 
Cox, M., Elen, J., Steegen, A. (2019) / Students' reasoning processes while constructing causal....

complex systems on different scales such as global climate change and migration flows. Furthermore, several practical implications for future design were mentioned such as offering the variables on paper strips or using software to facilitate the spatial organization of the variables in the diagram, the importance of carefully using specific words in information sources and as a variable label, and spending attention to a starting point for students in the constructing of the diagram.

\section{References}

Assaraf, O. B. Z., \& Orion, N. (2005). Development of system thinking skills in the context of earth system education. Journal of Research in Science Teaching, 42(5), 518-560. https://doi.org/10.1002/tea.20061

Biggs, J. B., \& Collis, K. F. (1982). Evaluating the quality of learning : the SOLO taxonomy (Structure of the observed learning outcome). New York: Academic Press.

Bruillard, E., \& Baron, G.-L. (2000). Computer-based concept mapping: a review of a cognitive tool for students. Proceedings of Conference on Educational Uses of Information and Communication Technologies, (august), 331-338. https://doi.org/10.1.1.365.3379

Collins, A., Brown, J., \& Newman, S. (1989). Cognitive apprenticeship: Teaching the craft of reading, writing, and mathematic. Knowing; Learning and Instruction: Essays in Honour of Robert Glaser, 453-494. https://doi.org/10.1017/CBO9781107415324.004

Collins, a., Brown, J. S., \& Holum, A. (1991). Cognitive apprenticeship: making thinking visible. American Educator, 15(3), 6-11, 38-46.

Cox, M., Elen, J., \& Steegen, A. (2017). Systems thinking in geography: Can high school students do it? International Research in Geographical and Environmental Education, O(0), 1-16. https://doi.org/10.1080/10382046.2017.1386413

Dogusoy-Taylan, B., \& Cagiltay, K. (2014). Cognitive analysis of experts' and novices' concept mapping processes: An eye tracking study. Computers in Human Behavior, 36, 82-93. https://doi.org/10.1016/j.chb.2014.03.036

Dörner, D. (1986). Diagnostik der operativen Intelligenz [Assessment of operative intelligence]. Diagnostica, 32 (4), 290-308.

Ericsson, K. A. (2006). Protocol Analysis and Expert Thought: Concurrent Verbalizations of Thinking during Experts ' Performance on Representative Tasks. In K. A. Ericsson, N. Charness, P. J. Feltovich, \& R. R. Hoffman (Eds.), The Cambridge Handbook of expertise and expert performance (pp. 223-241). Cambridge: Cambridge University Press. https://doi.org/https://doi.org/10.1017/CBO9780511816796

Ericsson, K. A., \& Simon, H. A. (1998). How to study thinking in everyday life: Contrasting think-aloud protocols with descriptions and explanations of thinking. Mind, Culture, and Activity, 5(3), 178-186. https://doi.org/10.1207/s15327884mca0503

Esters, I.G. \& Ittenbach, R.F. (1999) Contemporary theories and assessments of intelligence: A primer. Professional School Counceling, 2(5), 373-376

Favier, T. T., \& van der Schee, J. A. (2014). The effects of geography lessons with geospatial technologies on the development of high school students' relational thinking. Computers and Education, 76, 225-236. https://doi.org/10.1016/j.compedu.2014.04.004

Fischer, A., Greiff, S., \& Funke, J. (2012). The process of solving complex problems. The Journal of Problem Solving, 4(1), 19-42. https://doi.org/10.7771/1932-6246.1118 
Forrester, J. W. (1994). System dynamics, systems thinking, and soft OR. System Dynamics Review, 10(2-3), 245-256. https://doi.org/10.1002/sdr.4260100211

Funke, J. (2001). Dynamic systems as tools for analysing human judgement. Thinking \& Reasoning, 7(1), 69-89. https://doi.org/10.1080/13546780042000046

Funke, J. (2010). Complex problem solving: A case for complex cognition? Cognitive Processing, 11(2), 133-142. https://doi.org/10.1007/s10339-009-0345-0

Hmelo-Silver, C. E., Liu, L., Gray, S., \& Jordan, R. (2015). Using representational tools to learn about complex systems: A tale of two classrooms. Journal of Research in Science Teaching, 52(1), 6-35. https://doi.org/10.1002/tea.21187

Hogan, K., \& Thomas, D. (2001). Cognitive comparisons of students' systems modeling in ecology. Journal of Science Education and Technology, 10(4), 319-345. https://doi.org/10.1023/A:1012243102249

Horn, K. L., \& Cattell, R. B. (1966) Refinement and test of the theory of fluid and crystallized general intelligences. Journal of Educational Psychology, 57(5), 253-270

International Geographical Union. (2016). International charter on geographical education. Retrieved from http://www.igu-cge.org/wp-content/uploads/2018/02/IGU_2016_def.pdf

Jeong, A. (2014). Sequentially analyzing and modeling causal mapping processes that support causal understanding and systems thinking. In D. Ifenthaler \& R. Hanewald (Eds.), Digital Knowledge Maps in Education: Technology-Enhanced Support for Teachers and Learners (pp. 239-251). New York: Springer. https://doi.org/10.1007/978-1-4614-31787_13

Jonassen, D. H. (2004). Learning to solve problems: An Instructional design guide. San Francisco: Pfeiffer.

Jonassen, D. H., \& Henning, P. (1996). Mental models : Knowledge in the head and knowledge in the world. Technology, 39(3), 37-42. Retrieved from http://www.jstor.org/stable/44428530

Karkdijk, J., van der Schee, J. A., \& Admiraal, W. F. (2018). Students' geographical relational thinking when solving mysteries. International Research in Geographical and Environmental Education. https://doi.org/10.1080/10382046.2018.1426304

Kirschner, P. A. (2002). Cognitive load theory: implications of cognitive load theory on the design of learning. Learning and Instruction, 12(1), 1-10. https://doi.org/10.1016/S09594752(01)00014-7

Landis, J. R., \& Koch, G. G. (1977). The measurement of observer agreement for categorical data. Biometrics, 33(1), 159-174. https://doi.org/10.2307/2529310

Leat, D. (1998). Thinking through geography. Cambridge: Chris Kington Publishing.

Leat, D., \& Nichols, A. (2000). Observing pupils' mental strategies: Signposts for scaffolding. International Research in Geographical and Environmental Education, 9(1), 19-35. https://doi.org/10.1080/10382040008667627

Löhner, S., Van Joolingen, W. R., Savelsbergh, E. R., \& Van Hout-Wolters, B. (2005). Students' reasoning during modeling in an inquiry learning environment. Computers in Human Behavior, 21(3), 441-461. https://doi.org/10.1016/j.chb.2004.10.037

McAleese, R. (1994). A theoretical view on concept mapping. Research in Learning Technology, 2(1), 38-48. https://doi.org/10.3402/rlt.v2i1.9487 
Cox, M., Elen, J., Steegen, A. (2019) / Students' reasoning processes while constructing causal....

Ministerie van Onderwijs en Vorming. (2017). Sociale en technische wetenschappen - derde graad TSO. Retrieved March 15, 2018, Retrieved from https://www.onderwijskiezer.be/v2/secundair/sec_detail.php?detail=225\&var=3GTSO

Neisser, U., Boodoo, G., Bouchard, T.J., Boykin, A.W., Brody, N., Halpern, D.F., Loehlin, J.C., Perloff, R., Sternberg, R.J. \& Urbina, S. (1996) Intelligence: Knowns and unknowns. American Psychologist, 51(2), 77-101.

Newell, A., \& Simon, H. A. (1972). Human problem solving. Englewood Cliffs: Prentice-Hall.

Novak, J. D., \& Cañas, a J. (2008). The theory underlying concept maps and how to construct and use them. IHMC CmapTools. https://doi.org/Technical Report IHMC CmapTools 2006-01 Rev 2008-01

Öllinger, M., Hammon, S., von Grundherr, M., \& Funke, J. (2015). Does visualization enhance complex problem solving? The effect of causal mapping on performance in the computerbased microworld Tailorshop. Educational Technology Research and Development, 63(4), 621-637. https://doi.org/10.1007/s11423-015-9393-6

Senge, P. (1990). The Fifth Discipline. The art and practice of the learning organization. New York: Currency Doubieday. Retrieved http://www.wz.uw.edu.pl/pracownicyFiles/id10926-the-fifth-discipline.pdf

Shin, N., Jonassen, D. H., \& McGee, S. (2003). Predictors of well-structured and ill-structured problem solving in an astronomy simulation. Journal of Research in Science Teaching, 40(1), 6-33. https://doi.org/10.1002/tea.10058

Sins, P. H. M., Savelsbergh, E. R., \& van Joolingen, W. R. (2005). The difficult process of scientific modelling: An analysis of novices' reasoning during computer-based modelling. International Journal of Science Education, 27(14), 1695-1721. https://doi.org/10.1080/09500690500206408

Stratford, S. J., Krajcik, J., \& Soloway, E. (1998). Secondary students' dynamic modeling processes: analyzing, reasoing about, synthesizing, and testing models of stream ecosystems. Journal of Science Education Technology, 7(3), 215-234. https://doi.org/10.1023/A:1021840407112

Van Merriënboer, J. J. G., \& Sweller, J. (2005). Cognitive load theory and complex learning: Recent developments and future directions. Educational Psychology Review (Vol. 17). https://doi.org/10.1007/s10648-005-3951-0

Weinstein, C. F., \& Mayer, R. F. (1986). The teaching of learning strategies. In M. C. Wittrock (Ed.), Handbook of Research on Teaching (Third edit, pp. 315-327). New York: Macmillan Publishing Company.

White, B. Y., Shimoda, T. A., \& Frederiksen, J. R. (1999). Enabling students to construct theories of collaborative inquiry and reflective learning: Computer support for metacognitive development. International Journal of Artificial Intelligence in Education (IJAIED), 10(2), 151-182. https://doi.org/citeulike-article-id:4046742

\section{Biographical Statements}

Marjolein cox is a PhD student at the Department of Earth and Environmental Sciences at KU Leuven. Her PhD research focuses on systems thinking in geography in secondary education. 
Jan ELEN is a full professor at the Faculty of Psychology and Educational Sciences at KU Leuven in Belgium. His research is mainly in the field of instructional design with a focus on higher education and complex learning outcomes (e.g. critical thinking, solving ill-structured problems).

An STEegeN is an Assistant Professor in the Department of Earth and Environmental Sciences at the KU Leuven in Belgium. Her research interests focus on the way in which students of secondary schools, as well as students in geo-science programs (as geography and geology), learn geographical concepts. She has an interest in misconceptions, systems thinking, virtual field trips and STEM-education. 